Helbig, Marko; Witte, Herbert; Schack, Bärbel:

\title{
Zeitvariante Kreuzbispektralanalyse in der Biosignalanalyse
}

Zuerst erschienen in: Biomedizinische Technik = Biomedical Engineering. - Berlin [u.a.] : de Gruyter. - 48 (2003), S1, S. 184-185.

Jahrestagung der Deutschen, der Österreichischen und der Schweizerischen Gesellschaften für Biomedizinische Technik ; (Salzburg) : 2003.09.25-27

Erstveröffentlichung: 2003

Datum Digitalisierung: $\quad 2009-10-23$

ISSN (online): $\quad$ 1862-278X

ISSN(print) 0013-5585

DOI: $\quad$ 10.1515/bmte.2003.48.s1.184

[Zuletzt gesehen: 2019-12-12]

„Im Rahmen der hochschulweiten Open-Access-Strategie für die Zweitveröffentlichung identifiziert durch die Universitätsbibliothek IImenau."

"Within the academic Open Access Strategy identified for deposition by IImenau University Library."

„Dieser Beitrag ist mit Zustimmung des Rechteinhabers aufgrund einer (DFGgeförderten) Allianz- bzw. Nationallizenz frei zugänglich."

"This publication is with permission of the rights owner freely accessible due to an Alliance licence and a national licence (funded by the DFG, German

Research Foundation) respectively."

\section{DFG}

Nationallizenzen 


\title{
ZIIITVARIANTE KREUZBISPEKTRALANALYSE IN DER BIOSIGNALANALYSE
}

\author{
M. Ilclbig', H. Wittc', B. Schack ${ }^{2}$ \\ 'Institut für Biomedizinische Technik und Informatik, TU IImenau, Deutschland \\ 'Instılut fïr Medizinische Statistik, Informatik und Dokumentation, FSU Jena, Deutschland \\ E-Mail: Marko.Helbig(w)tu-ilmenau.de
}

SI:MIMIIRY An appropriate investigation of quadratic phasc couplings $\left.(Q)^{\prime} C^{\prime}\right)$ in non-stationary signals requires IIIIc-1 artint methods of bispectral analysis. The approach (1) 1 IImk-1 altant bispectral analysis based on the adaptively cciussucly estimated $3^{\text {nl }}$-order cumulants is generalised as : - - reder cross-cumulant estimation for time-variant crosshropcidral analysis. The ain of this study is the IIIs citigation of its functionality and its dynamical and stittstleal propertics in comparison with the known propertics of the (auto-)bispectral and bicoherence allalysis

\section{PINLEITUNG}

Mit Hilfe der Bispektralanalyse ist es möglich, yuadratische Phasenkopplungen zwischen 1 reyuenzkumponenten zu analysieren. Die aur ein festes l.itinteriall bezogene Bispektralanalyse setzt Signalanforderungen (vor allem Stationarität) voraus, denen reale Biosignale zumeist nicht genügen. In diesem Sinne sind für das Bispektrum besonders transient auftretende quadratische Phasenkopplungen hedeutsam. Daraus folgt, dass zur Analyse dieser sich zeitlich ändernden Phänomene auch eine zeitvariante Bispektralanalyse mit einer ausreichend hohen Auflösung im Spektralbereich erforderlich ist. Aus diesem Grunde wurde eine Methode der dynamischen Auto-Bispektralanalyse auf der Basis einer adaptiven rekursiven Schätzung der Kumulanten III. Ordnung entwickelt und in [1] vorgestellt.

In dieser Arbeit wird dicse Methodik zur zeitvarianten Kreuz-Bispektralanalyse und ihrer normierten Form, der Kreuz-Bikohärenzanalysc erweitert und verallgemeinert. Ziel ist es, die Funktionsfähigkeit dieser crweiterten Methode zu testen und Erfahrungen $\mathrm{zu}$ sammeln, um sie in einer anschließenden Studie sicher antvenden zu können.

\section{METHODEN}

Es seien $X=\left\{x^{(\prime)}(k)\right\}_{k=0,1,2, \ldots,} \quad Y=\left\{y^{(i)}(k)\right\}_{k=0,1,2, \ldots}$ und $Z=\left\{z^{(i)}(k)\right\}_{k=0,1,2, \ldots .}$ Zeitreihen dreier Signale. Der in [1] vorgestcllte Algorithmus der adaptiven rekursiven Kumulantenschätzung III. Ordnung beinhaltet die adaptive rekursive Mittelwertschätzung einer momentanen Kumulantenfunktion. Dieser Ansatz wird in dieser Arbeit zur adaptiven rekursiven Mittelung einer momentanen Kreuz-Kumulantenfunktion ICCF (instantaneous cross-cumulant function) verallgemeinert $(1,2)$. Die anschlicßende 2-dimensionale Fouriertransformation ergibt eine Sequenz zeitvarianter Bispektren (3). Im Falle getriggerter Datensätze können die zeitgleichen dynamischen Bispektren noch über alle Realisierungen gemittelt wèrden (4).

Um numerische Effekte bei der zeitvarianten KreuzBikohärenzschätzung zu vermeiden und einen numerisch stabilen Wertebereich zwischen 0 und $1 \mathrm{zu}$ garantieren, wird analog zur Auto-Bikohärenzanalyse der in [2] vorgestellte Normierungsalgorithmus verwendet.

$$
\hat{b}_{x y z}\left(\omega_{1}, \omega_{2}, k\right)=\frac{\left|\left\langle B_{x y z}^{(i)}\left(\omega_{1}, \omega_{2}, k\right)\right\rangle\right|}{\sqrt{\left\langle\left. B_{x y z}^{(i)}\left(\omega_{1}, \omega_{2}, k\right)\right|^{2}\right\rangle}}
$$

Wird nur die reine Phasenbeziehung (Biphase) betrachtet, so resultiert die Kreuz-Phasenbikohärenz:

$$
\begin{aligned}
& \hat{\Gamma}_{x y z}\left(\omega_{1}, \omega_{2}, k\right)=\left\langle\exp \left(j \varphi_{x y z}^{(i)}\left(\omega_{1}, \omega_{2}, k\right)\right)\right\rangle \\
& \text { mit: } \quad \varphi_{x y z}^{(i)}\left(\omega_{1}, \omega_{2}, k\right)=\arctan \left(\frac{\operatorname{Im}\left[B_{x y z}^{(i)}\left(\omega_{1}, \omega_{2}, k\right)\right]}{\operatorname{Re}\left[B_{x y z}^{(i)}\left(\omega_{1}, \omega_{2}, k\right)\right]}\right)
\end{aligned}
$$

mit $i=1,2, \ldots, N, k=0,1,2, \ldots, M-1$ und $s=\min \left\{k, k+\tau_{1}, k-\tau_{2}\right\}$ 


\section{ERGEBNISSE}

Die Untersuchungen zur Funktionalität der vorgestellten Methode zeigen identische dynamische und statistische Eigenschaften wie die der Auto-Bispektralanalyse.

Unterschiede bestehen lediglich im notwendigen Berechnungsaufwand und dem Interpretationsgehalt der resultierenden Matrizen. Bei der Kreuzbispektralanalyse kann nicht davon ausgegangen werden, dass die gekoppelten Frequenzkomponenten in allen drei Signalen gleichermaßen enthalten sind. Die bekannten Symmetrieeigenschaften des Auto-Bispektrums gelten deshalb beim Kreuz-Bispektrum nicht. Aus diesem Grunde müssen im Falle zweier Eingangssignale $x, y$ mindestens drei der sechs in Abb. 1 gekennzeichneten Bereiche berechnet werden. Im Falle dreier Eingangssignale $x, y, z$ müssen alle sechs Symmetriefelder berechnet werden, um über $B_{x y:}^{(i)}\left(\omega_{1}, \omega_{2}, k\right)=B_{x y z}^{*(i)}\left(-\omega_{1},-\omega_{2}, k\right)$ auf die gesamte Bispektrum-Matrix schließen zu können.

Je nach dem, welche der quadratisch phasengekoppelten Frequenzen in $\mathrm{x}$ und / oder $\mathrm{y}$ und / oder $\mathrm{z}$ enthalten sind, wird die Kopplung als Peak in einem oder mehreren bestimmten Symmetriefeldern widergespiegelt. Somit kann auch umgekehrt aus der Position des/der Peaks im Kreuz-Bispektrum auf das die entsprechende(n) Frequenz(en) enthaltende Signal geschlossen werden: 1], [: $f_{x}=f_{1}, f_{y}=f_{2}, f_{z}=f_{1}+f_{2} ; 3$, 4: $f_{x}=f_{1}, f_{y}=-f_{2}, f_{z}=f_{1}+f_{2}$ und 5 , G: $f_{x}=f_{1}, f_{y}=-f_{2}, f_{z}=-f_{1}-f_{2}$. Für die Bikohärenz- und Phasenbikohärenzmatrizen gelten diese Symmetrien in analoger Weise. Somit ist wesentlich, in welcher Reihenfolge die Eingangssignale in den Berechnungsalgorithmus eingehen. Das folgende Simulationssignal verdeutlicht dies.

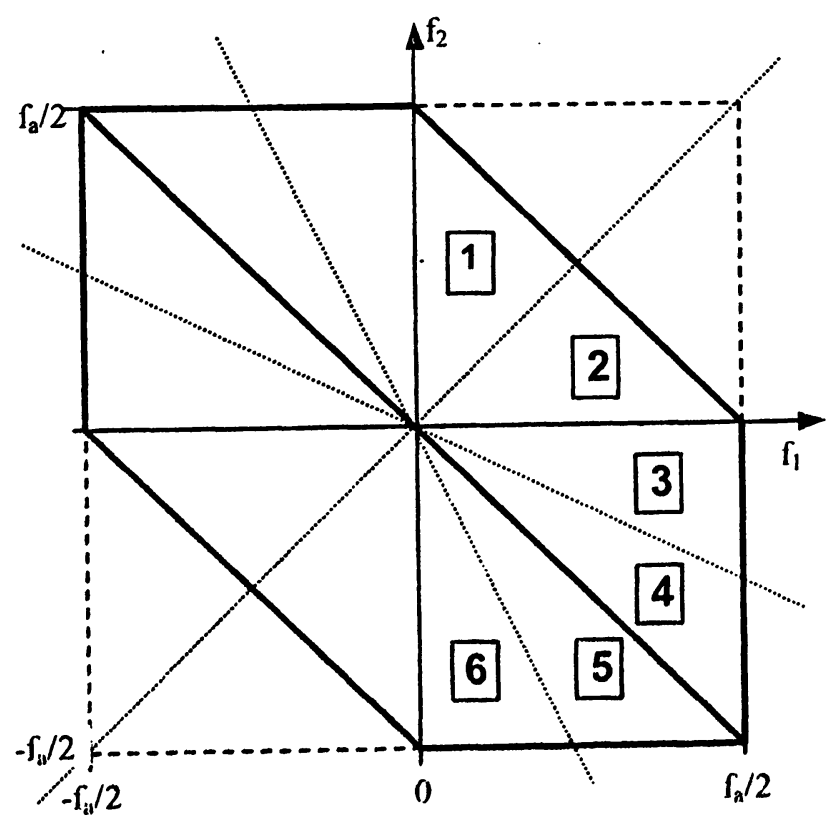

Abbildung 1: Symmetricberciche des Kreuz-Bispektrums
Jedes der Signale $x(k), y(k)$ und $z(k)$ beinhalte $u$. a. die Frequenzkomponenten $\mathrm{f}_{1}=3 \mathrm{~Hz}, \quad \mathrm{f}_{2}=7 \mathrm{~Hz}$ und $\mathrm{f}_{3}=\mathrm{f}_{1}+\mathrm{f}_{2}=10 \mathrm{~Hz}$. Während alle drei Auto-Bikohärenzen keine signifikanten Werte liefern, zeigt die KreuzBikohärenz $\hat{b}_{x y z}$ einen signifikanten Peak im Symmetriefeld 3. Daraus kann abgeleitet werden, dass eine quadratische Phasenkopplung zwischen $f_{1}$ von $y(t)$, $f_{2}$ von $z(k)$ und $f_{3}$ von $x(k)$ besteht. Alle anderen Varianten möglicher quadratischer Phasenkopplungen zwischen $x, y$ und $z$ erweisen sich als nicht existent.

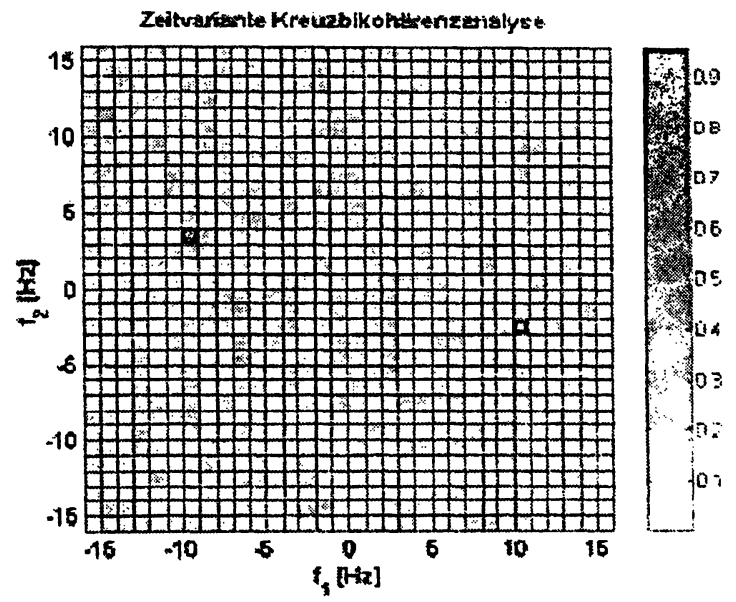

Abbildung 2: Kreuzbikohärenz des Simulationssignals

\section{SCHLUSSFOLGERUNGEN}

Die bewährte Methode der zeitvarianten Bispektralanalyse auf der Basis der adaptiven rekursiven Kumulantenschätzung konnte erfolgreich und oline Einschränkungen auf die KreuzBispektralanalyse ausgebaut werden. Die Effizienz des Einsatzes adaptiver rekursiver Schätzungen. insbesondere als Basisalgorithmus zur Analyse fortlaufender Signale, konnte auch anhand dieser komplexen Kenngrößen bestätigt werden.

Die hier vorgestellte und getestetc Methode zur zeitvarianten Kreuz-Bispektral- und Bikohärenzanalỵss wird in einer folgenden Studic angewendet, um Phasenkopplungsphänomene zwischen Frequenzkomponenten im EMG und MEG von ParkinsonPatienten mit Ruhe-Tremor hoch aufgelöst ?u analysieren.

\section{LITERATURHINWEISE}

[1] Helbig, M.. Gricßbach, (i., Schach. R., Wille, H ,Application of time-variant bispcetrum III biosignal analysis", Mcd. \& Biol. Fing. \& compul . 37, Supplement 2, 392-343, 10004

[2] Helhig. M., (iricßbach, (i., Witle, 11, Silhath. I „Numerisch stabile Normorung dis induch geschïzten Bispehtrums". Biomedi, Ims he Technik, 46 (lergainzungsband 1), 31s. 310. 21101

Diese Arbeit wurde geförden durch des PFli Prolich (iR 1555/2-3 und WI 1160.?.3. 